\title{
Aplicación de un nuevo sistema de indización en una colección de recursos especializados en ciencias de la educación
}

\author{
Por Mariàngels Granados y Anna Nicolau
}

\begin{abstract}
Resumen: Este estudio sobre una colección de ocho documentos sobre enseñanza primaria (ciencias de la educación) sirve de marco para presentar un nuevo sistema de indización, basado en una estructura de descriptores primarios y secundarios y su equivalencia con la Clasificación Decimal Universal. Se trata de poner de relieve las ventajas de su uso en dos vertientes: desde la perspectiva del sistema de búsqueda de la información y su implicación en el sistema y proceso de indización. Las directrices que rigen en este caso práctico explican el sistema de indización postcoordinado empleado en base a la técnica de ordenación nuclear de los descriptores. Para la gestión de la información en el contexto marcado, se plantea la necesidad de un sistema informático, del que también se indican una serie de requisitos que faciliten la explotación de sus posibilidades deconsulta y recuperación de información relevante.
\end{abstract}

Palabras clave: Lenguajes de indización postcoordinados, Clasificación Decimal Universal, Indización, Enseñanza primaria, Ciencias de la educación, Tesauros, Sistemas de clasificación, Catálogos en línea, Gestión de la información, Sistemas de recuperación de la información.

Title: Implementation of a new indexing system for a specialized resource collection in teacher preparation

Abstract: A collection of eight documents on primary education provides the example for presenting a new indexing system based on a structure of primary and secondary descriptors and illustrating its equivalence to Universal Decimal Classification. The article shows the advantages of this new system as an information retrieval system and describes its role in the indexing system and process. Another aim of this article is to describe an actual project using a postcoordinate indexing system based on nuclear ordering of the descriptors, as well as to define the system and software requirements for efficient operation of user search and information retrieval functions.

Keywords: Postcoordinate indexing languages, Thesauri, Classification systems, Universal Decimal Classification, Content analysis, Web catalogs, Information management, Information retrieval systems, Education.

Granados, Mariàngels; Nicolau, Anna. “Aplicación de un nuevo sistema de indización en una colección de recursos especializados en ciencias de la educación”. En: El profesional de la información, 2007, noviembre-diciembre, v. 16, n. 6, pp. 627-635.

DOI: 10.3145/epi.2007.nov.09

\section{Introducción}

ESTE TRABAJO CONSTA DE DOS PARTES: En primer lugar hemos situado el contexto de la colección de referencia que se ha indizado y resumido, especializada en ciencias de la educación y con diferentes tipos documenta- les. Hemos seleccionado este tema por nuestro conocimiento de la disciplina a nivel de indización y clasificación.

En esta primera parte se ha seguido el siguiente método: la descripción del sistema de indización piloto que aplicaríamos a los des- criptores y el análisis de contenido y selección de los términos de algunos documentos para comprobar su funcionamiento. Así hemos constatado que el método era idóneo y hemos continuado explicando los tesauros utilizados. Se estipulan unas concreciones sobre el uso y orden 
de los elementos y del sistema de clasificación aplicado $(C D U)$.

En la segunda parte del artículo se tratan las características que precisa el sistema informático de gestión documental para hacer posible el tratamiento y la recuperación de la información de acuerdo con las premisas establecidas.

\section{Contexto}

El perfil de centro del caso práctico es uno de carácter especializado en ciencias de la educación del grado educativo de primaria, secundaria y bachillerato en España, cuyos objetivos serían: la investigación educativa, la psicología y sociología de la educación, la formación del profesorado, la dinamización pedagógica, el apoyo a la docencia y las actividades preescolares. La colección incluye documentos de diferentes tipos según criterios tales como sus características físicas, el contenido o su naturaleza (original, copias impresas o seriado). La audiencia prevista en una unidad de información de tales características tiene en cuenta el profesorado, investigadores y profesionales de áreas de especialidad afines como pedagogos, psicólogos, trabajadores sociales y educadores.

Si bien el centro en base al cual hemos efectuado las indizaciones es especializado, tenemos que remarcar el ámbito multidisciplinar en el que ubicamos la recuperación de la información según el sistema expuesto.

\section{Sistema de indización}

\subsection{Análisis}

En el proceso de indización la coherencia es un factor clave, puesto que la subjetividad condiciona la selección de descriptores por parte de un mismo profesional en distintos momentos y más incluso cuando se trata de la misma catalogación realizada por diferentes analistas. Aunque se pretenda alcanzar una buena política de indización, el nivel de coherencia nunca suele ser tan elevado como nos gustaría. En EUA, este porcentaje se sitúa en el $60 \%$ como máximo, aunque habitualmente es del 25\%. Así, esta incoherencia deja de ser una anomalía y pasa a ser un hecho normal. Con frecuencia, el grado de coherencia es muy alto en los dos primeros términos seleccionados, motivo por el cual acostumbra a coincidir con los conceptos principales.

\subsection{Sistema de indización}

Nuestro planteamiento de nuevo sistema de indización está basado en la utilización de descriptores y de la Clasificación Decimal Universal $(C D U)$. La $C D U$ resulta ser el equivalente o la transliteración de un sistema de descriptores primarios asignados de una manera lógica y ordenada con el fin de representar el contenido principal del documento.

Las ventajas de este nuevo sistema quedan dibujadas por lo que denominamos "índices permutados" de descriptores y de la $C D U$. Estos índices son los más adecuados para evidenciar la sistematización de este proceso que relaciona los temas y números de las notaciones decimales, gracias a la frecuencia de uso en generarse, y que supone una garantía para solucionar algunos de los principales problemas de la indización en el mundo bibliotecario, como la coherencia, la selectividad o la especificidad y la uniformidad, conceptos que explicaremos más adelante.

Además, los índices facilitan la comparación de los términos con las signaturas correspondientes a un mismo concepto por su proximidad, y a la vez representan la frecuencia en la que han sido asignados y combinados por parte de los profesionales durante el proceso de catalogación. Esta frecuencia permite también traslucir la correspondencia (que en un índice real se hace patente de forma más clara) entre las materias y los códigos numéricos elaborados con la clasificación decimal. Así pues, se puede saber si un conjunto de descriptores se ha utilizado junto con una misma notación para representar idéntico concepto. Y es este hecho el que proponemos marcar como pauta a seguir (en relación a los principios de procedimiento que antes hemos mencionado), dado que siempre se procurará actuar del mismo modo. En este caso podemos demostrar la potencia de este sistema de indización para conseguir la máxima coherencia posible. La uniformidad del catálogo queda entonces garantizada puesto que el contenido de los documentos que tratan de los mismos conceptos se indizará con los mismos términos controlados.

\section{"El proceso descrito permitirá controlar y ordenar todos los conceptos que expresen un determinado contenido de manera lógica y será siempre exhaustivo"}

Mediante la correspondencia del conjunto de descriptores con la $C D U$ también conseguimos un alto grado de especificidad. No habrá ningún conjunto de descriptores primarios que representen un nivel más general ni más específico que el que represente la notación asignada. El proceso descrito permitirá controlar y ordenar todos los conceptos que expresen un determinado contenido de manera lógica y será siempre exhaustivo. No obstante, la política del centro dónde se aplique deberá marcar su uso en mayor o menor grado en función de la colección, necesidades y objetivos. La correlación en línea de materias y notaciones de clasificación podría ayudar a normalizar y conseguir la uniformidad esperada. 
En relación a la recuperación, la búsqueda por materia o palabra clave nos llevaría a identificar su número correspondiente, como siguiente paso. La equivalencia mencionada se obtendría de la permutación de los índices de encabezamientos de materia a $C D U$, y también de $C D U$ a encabezamientos de materia recíprocamente. Estos índices ponen de manifiesto las interacciones de los lenguajes documentales empleados y permiten ver la frecuencia de uso de la notación de todos los documentos de la base de datos.

El procedimiento en cadena ideado por Ranganathan $^{1}$ ha sido muy estudiado y desarrollado en la actualidad: una de las especialistas es Rosa San-Segundo de la Universidad Carlos III de Madrid, que tiene extensa bibliografía publicada sobre la indización y la clasificación en cadena. Nuestro estudio se fundamenta en esta técnica, pero además de coherencia pretende conseguir un nivel de relevancia más alto mediante la ponderación de los descriptores al separar en dos los campos de descriptores primarios y los secundarios (los tratados con menor intensidad o en parte del documento) y darles un orden lógico.

\subsection{Tesauro}

En la colección de referencia especializada en ciencias de la educación, hemos optado por una combinación de lenguajes postcoordinados: un tesauro que elaboraríamos adaptando los ya existentes en este campo a la realidad concreta:

- ERIC (Educational Resources Information Center): processing and reference facility, 2003.

http://www.eric.ed.gov/

- Houston, James E. Thesaurus of ERIC descriptors. 12th ed. Phoenix: Oryx, 1990.

- Comissió de les Comunitats Europees, i Consell d'Europa. Tesaurus europeu de l'educació. Versió en llengua catalana, 2003. http://www.mec.es/redinet2/ html/TEECAT.pdf

ERIC es un sistema de información patrocinado por el Departamento de Educación de los EUA $\mathrm{y}$ el Institute of Education Science. En nuestro estudio seguiremos este tesauro como fuente de referencia (previa traducción y adaptación al contexto). Hemos consultado la versión en línea de 2003 en combinación con la impresa del año 1995. La base de datos que utiliza este lenguaje estructura la información de la forma que puede verse en la figura 1.

Los descriptores precedidos por un asterisco son primarios ("Major descriptors") y el resto son secundarios ("Minor descriptors") (ERIC, 1995). Los identificadores, como veremos, se ofrecen en otro campo al igual que el tipo de documento cuando no es materia. En cuanto a los descriptores observamos que están todos mezclados sin ningún orden aparente. Nuestra base de datos, en cambio, dispondría la información como puede verse en la figura 2.

Según el ejemplo, éstos serían los términos de indización correspondientes a un documento que nos hable principalmente de propuestas

High School Foreign Language Programs: A renewed Challenge.

Descriptors: Secondary Education; *Second Language

Instruction; *Cultural Awareness; Cultural Education; Critical

Thinking; Worksheets; Cross Cultural Studies; Reading Instruction; German

Identifiers: Cultural Literacy

Pub Type: didácticas y de actualización científica sobre aritmética para maestros y ofrece como contexto la reforma educativa en Catalunya haciendo referencia sólo superficialmente a la italiana. Como veremos, el sistema informático que emplearemos también tendrá que permitir realizar la búsqueda según su relevancia. $\mathrm{Si}$ tenemos en cuenta la visualización de la descripción del documento, los diferentes elementos siguen un orden y el registro reúne en un mismo campo descriptores e identificadores.

Esta disposición no responde al orden en que aparecen en el documento, sino al orden "nuclear" de los conceptos principales o más relevantes que expresan los documentos. Para los descriptores secundarios habrá otro campo que se creará siguiendo el criterio expuesto.

Se ha considerado por orden de relevancia indicar los identificadores al final de la secuencia, concretamente los geográficos y cronológicos. Sin embargo, esto no impide que todos los términos queden indizados separadamente y ofrezcan el mismo potencial de búsqueda:

- Descriptores extraídos de los lenguajes controlados citados anteriormente.

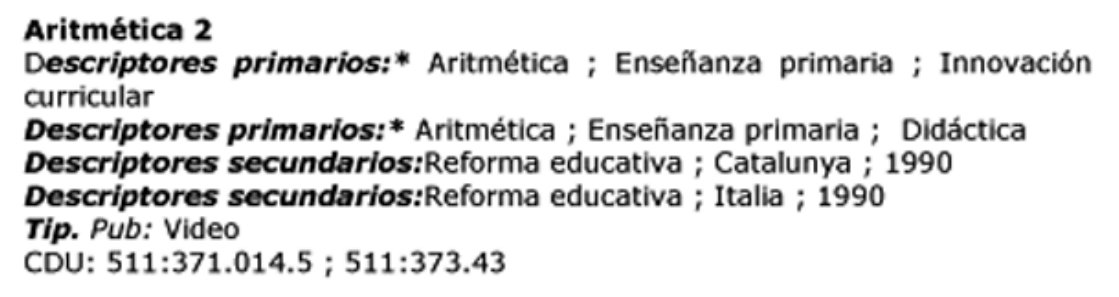

Figura 2 


\begin{tabular}{|c|c|}
\hline 37.014.6(467.1 Mr Alella) & Escuela pública; Evaluación; Alella (Catalunya); 1998 \\
\hline 37.057CEIP Fabra(467.1 Mr Alella) & Escuela pública; CEIP Fabra; Alella (Catalunya); 1998 \\
\hline $371: 681.3(467.1)$ & Enseñanza; Nuevas tecnologías; Catalunya \\
\hline 371.014.1(467.1 Ba Badalona)"1971" & $\begin{array}{l}\text { Enseñanza primaria; Igualdad de oportunidades; Badalona } \\
\text { (Catalunya); } 1971\end{array}$ \\
\hline 371.014.53(467.1 Ba Badalona)"1971" & Enseñanza primaria; Desigualdad social; Badalona; 1971 \\
\hline $371.12: 681.3(467.1)$ & Profesión docente; Red telemática; Catalunya \\
\hline 371.263(467.1 Ba Badalona)"1971" & $\begin{array}{l}\text { Enseñanza primaria; Evaluación inicial; Tests de diagnóstico; } \\
\text { Badalona (Catalunya) ;1971 }\end{array}$ \\
\hline $373.3 / .47(467.1)$ & Enseñanza primaria; Catalunya \\
\hline $373.3(094.58)(460) " 18^{\prime \prime}$ & $\begin{array}{l}\text { Enseñanza primaria; Sistema educativo; Legislación; } \\
\text { España; XIX }\end{array}$ \\
\hline $373.3 .078(467.1)$ & $\begin{array}{l}\text { Enseñanza primaria; Escuela pública; Administrador; } \\
\text { Catalunya }\end{array}$ \\
\hline $511: 371.014 .5$ & Aritmética; Enseñanza primaria; Innovación curricular \\
\hline $511: 373.43$ & Aritmética; Enseñanza primaria; Didáctica \\
\hline 78:371.3Mètode Willems i Chapui & Método Willems y Chapui; Enseñanza primaria \\
\hline $78: 373.3 .02$ & Educación musical; Enseñanza primaria \\
\hline 804.99:373.3(467.1 Ba Badalona)"1971" & $\begin{array}{l}\text { Lengua catalana; Enseñanza primaria; Badalona } \\
\text { (Catalunya); } 1971\end{array}$ \\
\hline
\end{tabular}

Tabla 1. Ejemplo de índice permutado de CDU/descriptores.

Los diferentes descriptores quedan ordenados según el orden decimal del 0 al 9.

- Identificadores para representar y designar nombres de personas y entidades, geográficos, períodos de tiempo y las tipologías documentales.

\section{Sistema de clasificación}

Para la clasificación documental se sigue la versión expandida de la $C D U$, en este caso la subclase 37 :

- Universal Decimal Classification (BSI Standards). English full ed. London: British Standards Institution, 1975.

Cuando haga falta optar por la relación de notaciones, se recurrirá a la edición abreviada española:

- CDU: Clasificación Decimal Universal. Madrid: Aenor, 1995.

Para subdivisiones geográficas de topónimos catalanes:

- Rubió i Balaguer, Jordi, dir. Classificació decimal per a les biblioteques catalanes. Barcelona: Teide, 1982.
Los argumentos que podemos aportar para justificar el importante papel de las clasificaciones en la recuperación de la información en sistemas automatizados vienen a ser los que se listan a continuación:

- Capacidad de combinar la búsqueda por palabra clave (recuperación) con la búsqueda por índice (navegación). Los sistemas de clasificación permiten la visualización por pantalla de los registros temáticamente afines. Además, el esquema puede ser utilizado como posible interfaz de cara a la consulta general de un catálogo por materias (por ejemplo, la Biblioteca Virtual Cervantes).

- Contextualización de las búsquedas cubiertas por un campo semántico. Este aspecto resulta especialmente práctico cuando se combina con la recuperación por palabras clave a la hora de reducir los problemas de ambigüedad del lenguaje natural.
- Modificar la estrategia de búsqueda, ampliándola o limitándola de un nivel más genérico a otro más específico gracias a la capacidad de navegación implícita de la organización jerárquica de la información, a partir del código resultante asignado a un registro bibliográfico conocido.

- Las notaciones pueden funcionar como un lenguaje puente para contrarrestar y sobrepasar barreras lingüísticas en bases de datos muy amplias y con registros en diferentes idiomas.

- La organización de los conceptos está basada en las relaciones de materia y no en el alfabeto. Esto hace que los temas dentro de una disciplina queden agrupados en lugar de quedar dispersos (utilizando un orden aleatorio o más bien convencional).

Las ventajas que se han valorado de la $C D U$ son además:

- La simplificación en la repre- 


\begin{tabular}{|c|c|}
\hline Aritmética; Enseñanza primaria; Innovación curricular & $511: 371.014 .5$ \\
\hline Educación musical; Enseñanza primaria & $8: 373.3 .02$ \\
\hline Enseñanza; Nuevas tecnologías; Catalunya & 371: $681.3(467.1)$ \\
\hline Enseñanza primaria; Evaluación inicial; Tests de diagnóstico; & 371.263(467.1Ba Badalona)"1971" \\
\hline Badalona (Catalunya); 1971 Enseñanza primaria; Catalunya & $373.3 / .47(467.1)$ \\
\hline Enseñanza primaria; Desigualdad social; Badalona; 1971 & 371.014.53(467.1Ba Badalona)"1971" \\
\hline $\begin{array}{l}\text { Enseñanza primaria; Escuela pública; Administrador; Cata- } \\
\text { lunya }\end{array}$ & $373.3 .078(467.1)$ \\
\hline \multicolumn{2}{|l|}{ Enseñanza primaria; Igualdad de oportunidades; } \\
\hline Badalona & 371.014.1(467.1Ba Badalona)"1971" \\
\hline \multicolumn{2}{|l|}{ (Catalunya); 1971} \\
\hline Enseñanza primaria; Sistema educativo; España; XIX & $373.3(094.58)(460)^{\prime \prime} 18^{\prime \prime}$ \\
\hline Escuela pública; Evaluación; Alella (Catalunya); 1998 & 37.014.6(467.1 Mr Alella) \\
\hline Escuela pública; CEIP Fabra; Alella (Catalunya); 1998 & 37.057CEIP Fabra(467.1 Mr Alella) \\
\hline \multicolumn{2}{|l|}{ Lengua catalana; Enseñanza primaria; } \\
\hline Badalona (Catalunya); 1971 & 804.99:373.3(467.1 Badalona)"1971" \\
\hline Método Willems y Chapui; Enseñanza primaria & 78:371.3Mètode Willems i Chapui \\
\hline Professió docent; Red telemática; Catalunya & $371.12: 681.3(467.1)$ \\
\hline
\end{tabular}

Tabla 2. Ejemplo de índice permutado de descriptores / CDU

El juego de descriptores primarios de los documentos quedan ordenados alfabéticamente.

sentación de los conceptos, como aplicación de una sintaxis para relacionar los términos.

- La construcción de notaciones equiparables a la cadena de descriptores.

- La flexibilidad con respecto a la combinación de elementos y a la ordenación de algunas subdivisiones para adaptarse al orden nuclear $\mathrm{y}$ a las necesidades locales.

- La cobertura universal de todas las materias, de manera más o menos desarrollada.

- La organización y visualización de manera que permite destacar las relaciones entre las diversas áreas temáticas.

- La polijerarquía, valorada comouna característica positiva de su estructura, pues la asignación de más de un término genérico a cada descriptor contribuiría a dar flexibilidad al lenguaje documental para representar los diferentes enfoques o puntos de vista sobre los cuales puede ser tratado un mismo tema.

- La organización de algunos conceptos por facetas (subdivisiones especiales).

El principal inconveniente que apuntan los teóricos ${ }^{2}$ y que hemos constatado en la utilización de la $C D U$ es la necesidad por parte del usuario de saber el número concreto que pertenece a su concepto $\mathrm{y}$, por lo tanto, la necesidad de conocer su estructura, su funcionamiento y el no disponer de una versión en línea actualizada y de acceso gratuito (UDC Consortium, en línea). Este problema se puede sortear si hacemos la primera consulta por descriptores mediante la estructura de navegación o bien por palabra clave ya que entonces los índices nos enviarán al número concreto de $C D U$, que a su vez nos servirá para ampliar la búsqueda a los conjuntos de descriptores primarios que sean equivalentes.
La opción complementaria consistiría en prescindir del índice temático de $C D U$ y utilizar enlaces de dos niveles: unos serian encabezamientos temáticos equivalentes a las notaciones de $C D U$, establecidos como clases y los otros serian los descriptores que representan el contenido de los documentos. En estos casos, el encabezamiento correspondiente a las clases y subclases debería diferenciarse tipográficamente del resto. Su objetivo: contrarrestar la dificultades para los usuarios derivadas del uso de la $C D U$ y contextualizar el usuario en el ámbito multidisciplinar correspondiente en cuanto al sistema de recuperación. De este modo, no sería imprescindible conocer los códigos y directamente el acceso se haría por medio de la información textual, por lo que entonces recurriríamos a las subdivisiones especiales de cada clase para una estructura facetada en la que se contemplarán 
entre otras ${ }^{3}$ los identificadores con connotación de tiempo, tipo documental, punto de vista y lugar.

Durante la elaboración del presente trabajo han surgido interrogantes sobre la visualización de los documentos con notaciones relacionadas. Este problema se ha sorteado con la previsión de la técnica de ubicación múltiple que hemos estipulado y creemos que debería tener el sistema de gestión documental. Otra cuestión planteada ha sido la gestión de las palabras clave con las que el usuario consulta el sistema. La decisión ha consistido en canalizarlas con los descriptores y encabezamientos afines consiguiendo así mediante estos enlaces (tanto de palabras clave como entre las clases establecidas) un amplio acceso a la información y facilitar la navegación, consideraciones que deberá contemplar el sistema informático.

\section{Sistema informático de gestión documental}

El sistema de recuperación de información (SRI) empleado debería ser integrado y escalable para que permitiera llevar a cabo todas las tareas de gestión bibliotecaria y de manera progresiva, por módulos; entre ellas: la catalogación y el control de autoridades, consulta (opac), adquisiciones, circulación (préstamo, reservas, prórrogas, etc.), control y seguimiento de las publicaciones periódicas, consulta del directorio de usuarios, edición y creación de estadísticas y de otros listados (por ejemplo, el de novedades o bibliografías para la difusión selectiva de la información, según perfiles de usuarios).

\subsection{Módulo de catalogación y indización}

A continuación se listan las posibilidades que ofrecerá y deberá garantizar el sistema:

- Admitir el formato MARC21 para la descripción bibliográfica $\mathrm{y}$ visualización de resultados.
- Dejar exportar o importar registros en este mismo formato.

- Ejercer control de los ejemplares: numeración a partir de códigos de barras y código topográfico.

- Copiar registros con la finalidad de crear otros registros nuevos.

- Diseñar varias plantillas de entrada de datos según modelos por tipologías de documentos.

- Corregir automáticamente una autoridad en todos los registros bibliográficos asociados a la vez.

- Indizar aquellos conceptos que necesiten de más de un término para ser representados (cadenas de descriptores): por ejemplo, nombreadjetivo con connotación diferente a la suma de los dos conceptos por separado.

- Ponderar los descriptores (descriptores primarios y descriptores secundarios).

- Hacer enlaces a documentos electrónicos/digitales relacionados: imágenes, ayudas de búsqueda, fuente, etc.

- Trabajar con diferentes catálogos que formen un todo (un catálogo formado por varios grupos o clases).

- Gestionar el tesauro para establecer referencias que relacionen los diferentes términos de indización y sin que generen incompatibilidades (referencias de véase, véase también y jerárquicas).

- Crear automáticamente índices de los campos: autor, título, materias (descriptores e identificadores), palabras clave y tipos de documentos. Aparte, poder definir el fichero de palabras vacías.

- Tener en cuenta los campos de: "Descriptores", "Identificadores", "CDU”y "Área temática” (notación/ nes equivalente/s a los conceptos nucleares y que podrían ser más de uno en los casos de polijerarquía o doble dependencia jerárquica) ${ }^{4}$.
- Hacer búsquedas postcoordinadas por los elementos de la $C D U$ que conforman las notaciones, sin necesidad de introducirla desdoblada (por separado) en el caso de las que estén relacionadas por medio del símbolo específico (:).

- Introducir en el sistema los sumarios sólo de las obras colectivas entendiendo como tales las compilaciones y las publicaciones periódicas especializadas: revistas, boletines, anuarios, jornadas, congresos y seminarios.

- Gestionar las autoridades de $C D U$.

\subsection{Módulo de consulta y re- cuperación}

Requisitos:

- El módulo de administración debe permitir modificar los índices para añadir, eliminar o corregir, una vez implementado el sistema.

- Integrar el control automático de autoridades con las relaciones jerárquicas y juegos de referencias pertinentes.

- Los lenguajes de indización/ recuperación deberán ser consultables para los usuarios de manera que puedan guiarlos y asistirlos durante el proceso de búsqueda por navegación: se requeriría el acceso al tesauro y a los índices permutados de materias y notaciones que definen el sistema.

- Poder visualizar todos los campos informativos empleados en las plantillas de entrada de datos, incluida la totalidad de las notas.

- Capacidad de navegación a partir de cada uno de los descriptores y las notaciones.

- Seleccionar los campos por los que se podrá consultar el catálogo: autor, título, materia, año, palabra clave, topográfico, etc.

- Implementar un motor de búsqueda interno en la base de da- 
tos que permita recuperar a texto completo y de forma ponderada sólo en los campos de título, resumen, identificadores y sumarios. Y también contemplar la opción de búsqueda avanzada por campos tal cual, en general, haciendo distinción entre descriptores primarios y secundarios con el fin de: extraer listados o índices de palabras clave a partir de los datos internos; hacer búsquedas utilizando los operadores booleanos; permitir el truncamiento de las palabras; eliminar o más bien prevenir, en definitiva, el ruido documental en los resultados.

- Recuperación a partir de todos los términos específicos de cada término genérico al cual están estos subordinados.

- Posibilidad de ampliar o limitar la búsqueda a términos más genéricos o específicos gracias a la capacidad de navegación implícita en la estructura jerárquica, a partir de los descriptores y viceversa.

- Los índices permutados (tablas de correspondencias entre descriptores y $C D U$ ) se generarían y permitirían la búsqueda a partir de los registros introducidos en ambas direcciones: por $C D U$ en orden jerárquico (con sus correspondientes descriptores) y por descriptores alfabéticamente por el primer elemento (con su correspondiente notación). En los registros quedaría reflejada esta información en los campos de "Clasificación" y "Descriptores". Así pues, en el primer caso ( $C D U$-descriptores), se podría buscar a partir de cada uno de los dos campos todos aquellos registros que tuviesen asociados, ordenándose de más generales a más específicos. En el segundo (descriptores$C D U)$, los grupos de descriptores en línea se ordenarían alfabéticamente por el primero de ellos, quedando a la vez relacionados y próximos teniendo en cuenta que su orden viene determinado por la importancia y novedad de los conceptos de más a menos (orden nuclear).
- Cuando las signaturas incluyan el signo de relación (:) se podrá buscar por los dos elementos. El enlace enviará el usuario a la posición del índice que se correspondería con el segundo elemento facilitando ver otros documentos relacionados: es decir, como opción alternativa de navegación.

- Posibilidad de consultar las notaciones de la $C D U$, destacadas tipográficamente, además de incluir las notaciones.

- Respecto a los descriptores e identificadores, cada uno de los elementos de ambos campos serían vinculados al tesauro y al índice alfabético de palabras clave, respectivamente. En este último caso, las palabras clave utilizadas en la búsqueda que se consideraran como referencias de descriptores aceptados en el tesauro, remitirían a éstos en base a la definición de los registros de autoridad.

- Se podrá navegar por el tesauro alfabéticamente, por orden jerárquico y por facetas que a su vez tendrán en cuenta las subdivisiones de la $C D U$ auxiliares generales así como también las específicas.

- Limitar la acción del buscador por palabras clave a determinados campos contribuiría a la recuperación de la información relativamente relevante (en función del sitio dónde el motor o robot buscará). Se trataría de un modelo inicial de búsqueda avanzada que se podría complementar con la búsqueda por campos, opción que excluiría el resumen; el resumen en el primer supuesto (la recuperación por palabra clave) sí que sería objeto de indización por parte de la aplicación.

- Presentar diferentes formatos y versiones de salida de las descripciones: mediante formulario o $I S B D$ (para los usuarios), en MARC (para el personal bibliotecario) y en versión reducida y detallada (según interés).
- Facilitar el acceso al catálogo vía web mediante una interfaz que se pueda parametrizar y sea fácil de usar.

- Reutilización de las búsquedas.

- Seleccionar de los registros obtenidos como resultado aquellos que interesen para un uso posterior.

- Exportar los resultados.

- Autoprotección frente a las consultas excesivamente largas y evitar la caducidad de las sesiones.

- La no interferencia de los acentos y del uso de mayúsculas/ minúsculas.

\section{Conclusiones}

Al llegar al final de este trabajo y después de aplicado el sistema de indización que se proponía en una colección representativa de ocho documentos especializados en ciencias sociales, más la experiencia profesional previa, podemos concluir que se ha comprobado su efectividad. En relación a los requisitos sugeridos para el programa informático de gestión, será conveniente que, en colaboración con el personal informático y según el margen o no de presupuesto disponible, se lleven a cabo las conversaciones, demostraciones $\mathrm{y}$ pruebas necesarias para establecer las pautas que permitan obtener un sistema lo más eficaz posible respecto a su diseño y a las posibilidades de recuperación de la información.

Queremos destacar, en relación a los procesos de indización y de recuperación, la importancia que adquiere la cadena lógica de descriptores (orden nuclear) al margen de que se puedan lanzar búsquedas a partir de cualquiera de los descriptores e identificadores que la conforman.

Según la bibliografía consultada, faltaría realizar más estudios sobre la elaboración de un formato 


\section{Ejemplo de registro y comentarios}

Bayés, Ramon and Ernest Garriga. "Repertorios conductuales mínimos en dos grupos de niños de diferente nivel socioeconómico". Anuario de psicología 5 (1971): 43-65.

\section{Tipo de documento:}

Se trata de un artículo que originariamente se publicó en una revista que editaba dos números al año, hasta 1989 en que la periodicidad se convirtió en trimestral. El documento que analizamos es el resultado de publicar de manera independiente el texto conservando su paginación: se considerará por tanto como separata.

\section{- Descriptores primarios}

\section{Educación}

Enseñanza primaria; Igualdad de oportunidades; Badalona (Catalunya); 1971

371.014.1(467.1 Ba Badalona)"1971"

\section{Educación}

Enseñanza primaria; Desigualdad social; Badalona; 1971

371.014.53(467.1 Ba Badalona)"1971"

\section{Educación}

Enseñanza primaria; Evaluación inicial; Tests de diagnóstico; Badalona (Catalunya) ;1971

371.263(467.1 Ba Badalona)"1971"

\section{Lenguas}

Lengua catalana; Enseñanza primaria; Badalona (Catalunya); 1971

804.99:373.3(467.1 Ba Badalona)"1971"

\section{- Descriptores secundarios}

Ley General de Educación (1970)

Inmigración; Badalona (Catalunya); 1971

\section{Resumen:}

El artículo es el resultado de la investigación realizada en enero y febrero de 1971 por los autores, con el objetivo de hacer un estudio comparativo entre dos muestras de población infantil al iniciarse el ciclo educativo (de 8 niños/niñas de 6-7 años cada una) en relación con el origen familiar, los factores socioeconómicos en el área urbana barcelonesa, concretamente de dos escuelas diferentes de Badalona y en relación al estudio de la lengua catalana. Con motivo de la aprobación de la Ley General de Educación (1970), se pretende demostrar que para garantizar la igualdad de oportunidades para acceder a la Educación
General Básica es preciso que todos los alumnos hayan conseguido un repertorio conductual básico de respuesta que en este caso se denomina instrumento BG: aptitudes y habilidades mínimas. Partiendo de la hipótesis que no se cumplen, se explica la metodología utilizada y se hace un análisis estadístico que acaba confirmándola. Se observa también que las diferencias sólo son significativas cuando se trata de las niñas.

\section{Contexto:}

- Los autores son profesores e investigadores universitarios catalanes $y$, en este documento, se complementan en tanto que se aplica la metodología científica en el ámbito de la psicología (aspectos sociales y que condicionan la conducta de los niños durante la etapa de educación primaria habiendo cursado al menos un año de formación preescolar) con los conocimientos estadísticos.

Ramon Bayés es Doctor en Filosofía y Letras (Sección de Psicología) por la Universidad de Barcelona y Diplomado en Psicología Clínica por la misma Universidad. Ha sido profesor. Desde finales de los años 70's se especializó en psicología de la salud y fue desde 1983 catedrático del Departamento de Psicología de la Educación en la Universitat Autònoma de Barcelona.

Ernest Garriga es titular del Departamento de Matemática Aplicada y Telemática de la Universitat Politècnica de Catalunya.

- Desde el punto de vista psicológico es interesante conocer el modelo conductual básico que establecen los autores para evaluar las muestras de población.

- Es importante para el estudio de la historia de la enseñanza primaria en Catalunya y España, por extensión, al final del período franquista. Un año más tarde de la implantación de la Ley General de Educación se describe la población de una ciudad catalana con mucha inmigración procedente del resto del estado y que presentaba diferencias respecto el nivel socioeconómico, de aprendizaje básico, etc. Por lo que tiene que ver con la política educativa del momento también interesa el uso del catalán (esta población no conocía la lengua catalana) y en la educación de las niñas las desigualdades eran más marcadas.

\section{Análisis de la indización}

Nos hemos basado en el planteamiento inicial (hipótesis) que se plantean los autores y hemos querido representar la totalidad de conceptos im- 
plícitos y explícitos del documento. Hemos definido en la política de indización, que sería una tipología de las que se tratarían a nivel más exhaustivo.

En el caso del concepto 2) se ha optado finalmente por "Desigualdad social" dado que hace referencia al ámbito y situación socioeconómica de los diferentes grupos sociales, mientras que el descriptor de ERIC era más general. Para complementarlo, se ha seleccionado "Igualdad de oportunidades" en tanto que es un término relacionado y el documento trata de las diferencias entre los dos grupos poblacionales desde el punto de vista de su acceso a la educación.

"Tests de diagnóstico", conviene precisar que finalmente nos hemos decidido por la forma en plural del descriptor ya que en el documento se habla explícitamente de un instrumento de análisis concreto elaborado por los autores y denominado "Instrument BG", una descripción del cual se adjunta en un anexo (se trata como materia este instrumento y no el tipo de documento como materia).

Para representar el concepto 3) se prefiere "Evaluación inicial" a "Evaluation utilization" porque es más específico.

"Educación" es el común denominador de la mayoría de descriptores primarios, pues el ámbito de estudio se trata de forma transversal.

Los conceptos 5) y 6) son parciales y sólo hacen referencia a una de las muestras de población estudiadas, por este motivo se han considerado secundarios. adecuado para el tratamiento de la $C D U$, así como estudiar otros modelos de interfaces que permitan al usuario una consulta más ágil y rápida.

En estos momentos en Europa se está avanzando en el uso de herramientas tradicionales acoplándolas a las nuevas tecnologías, pues ya existen experiencias basadas en el uso de la $C D U$ como sistema de recuperación.

Sería deseable y conveniente, así pues, realizar esfuerzos para, esta vez sí, tirar del carro y aprovechar las nuevas tecnologías para innovar y mejorar los sistemas de recuperación existentes.

\section{Notas}

1. Kashyap, Madan-Moham. "Likeness between Ranganathan's Postulations based approach to knowledge classification and entity relationship data modelling approach". En: Knowledge organization, 2003, v. 30, n. 1, pp. 1-19.

2. Bates, Marcia. "El context i la interacció han de ser elements bàsics en els sistemes de recuperació de la informació. Entrevista por Mario Pérez-Montoro y Jesús Gascón”. En: Item, 2006, n. 42, pp. 51-62.

3. Ver apartado 4.2 donde se hace referencia a la polijerarquía y los registros de la colección de documentos analizados.

\section{Bibliografía}

Amato, Carol J. The world's easiest guide to using the MLA. Westminster: Stargazer, 1999. ISBN 0-9643853-7-6.

Bates, Marcia. "El context i la interacció han de ser elements bàsics en els sistemes de recuperació de la informació. Entrevista por Mario Pérez-Montoro y Jesús Gascón”. En: Item, 2006 n. 42 , pp. $51-62$.

CDU: Clasificación Decimal Universal. Madrid: AENOR, 1995. ISBN 84-8143-019-6.

Comissió de les Comunitats Europees; Consel d'Europa. Tesaurus europeu de l'educació. Versió en llengua catalana, 2003. Consultado en 30-06-07

http://www.doredin.mec.es/documentos/TEECAT.pdf

ERIC (Educational Resources Information Center): processing and reference facility, 2003. Consultado en: 30-06-07.

http://www.eric.ed.gov/

Granados-Colillas, Mariàngels. Bibliografia sobre Joventut. Barcelona, 1989.

Granados-Colillas, Mariàngels; Orrit-Ambrosio, Dionis. "Informe presentat al Servei de Biblioteques i del Patrimoni Bibliogràfic de la Generalitat de Catalunya corresponent a la revisió del procés d'indexació d'aplicació a l'Hemeroteca Nacional de Catalunya". Barcelona, 16 de desembre de 1991

Granados-Colillas, Mariàngels; NicolauPayàs, Anna. "Indexació i resum de 9 documents: aplicació d'un nou sistema d'indexació multilingüe”. Barcelona, UB, curs 2003-2004.

Granados-Colillas, Mariàngels; NicolauPayàs, Anna. "La recuperació de la informació en els catàlegs en línia: l'ús de la Classificació decimal universal i la seva implicació en la indexació". En: $7^{\circ}$ Congreso del Capítulo Español de ISKO, 2005. Barcelona: Universitat de Barcelona. Departament de Biblioteconomia i Documentació, 2005, pp. 249-267.

Houston, James E. Thesaurus of ERIC descriptors. 12th ed. Phoenix: Oryx, 1990. ISBN 089774-788-7.

Kashyap, Madan-Moham. "Likeness between Ranganathan's Postulations based approach to knowledge classification and entity relationship data modelling approach". En: Knowledge organization, 2003, v. 30, n. 1, pp. 1-19.

Lancaster, Wilfrid; Pinto, María. Procesamiento de la información científica. Madrid: Arco Libros, 2001. ISBN 84-7635-485-1.

Rubió-Balaguer, Jordi, (dir.). Classificació decimal per a les biblioteques catalanes. Barcelona: Teide, 1982. ISBN 84-307-7331-2.

San-Segundo, Rosa. "Indización en cadena y su aplicación práctica". En: La representación y la organización del conocimiento en sus distintas perspectivas: su influencia en la recuperación de la información: actas el IV Congreso ISKO-España, 1999, pp. 53-57. ISBN 84-8138-435-6.

Slavic, Aida. "UDC implementation: from library shelves to a structured indexing language". En: Digital library of information science and technology, v. 33, n. 3, 2004. Consultado en: 30-06-06. http://dlist.sir.arizona.edu/661/

UDC Consortium. Outline of the UDC. Consultado en: 30-06-06.

http://www.udcc.org/outline/outline.htm

Universal Decimal Classification (BSI Standards). English full ed. London: British Standards Institution, 1975. ISBN 0-580-08626-7.

Mariàngels Granados, Anna Nico-
lau, Biblioteca de Catalunya
mgranados2@gmail.com
anicol@telefonica.net

